\section{The Design and Evaluation of a Mobile based Application to Facilitate Self-care for Pregnant Women with Preeclampsia during COVID-19 Prevalence}

\author{
Khadijeh Moulaei ${ }^{1 \oplus}$, Kambiz Bahaadinbeigy², Zahra Ghaf- \\ faripour ${ }^{3}$, Mohammad Mehdi Ghaemi ${ }^{4 * \odot}$
}

\begin{abstract}
Preeclampsia is one of the most common complications of pregnancy that is very difficult to control and manage during the outbreak of COVID-19. One way to control and manage this disease is to use self-care applications. Therefore, the aim of this study was to design and develop a mobile-based application to facilitate self-care for women, who suffer from pregnancy poisoning in the COVID-19 pandemic. This study was conducted in two stages: In the first stage, according to the opinion of $20 \mathrm{ob}-$ stetricians and pregnant women, a needs assessment was performed. In the second stage, based on the identified needs, the application prototype was designed and then evaluated. For evaluation, 20 pregnant women were asked to use the application for 10 days. QUIS questionnaire version 5.5 was used for evaluation. Descriptive statistics and mann-whitney test in SPSS software version 23 were used for data analysis. Out of the 66 information needs that were identified via the questionnaire, 58 were considered in designing the application. Features of the designed application were placed in 5 categories: User's profile, lifestyle, disease prevention and control, application capabilities and user's satisfaction. The capabilities of the application consist of introducing specialized COVID-19 medical centers, search for the location of medical centers and doctors' offices, drug management, drug allergies, self-assessment, stress reduction and control, nutrition and diet management, sleep management, doctor's appointment reminders, communication with other patients and physicians, application settings. Pregnant women rated the usability of the application at a good level. The designed application can reduce the anxiety and stress due to preeclampsia feel and also improve their knowledge as well as attitude towards the COVID-19 pandemic and preeclampsia.
\end{abstract} Citation: Moulaei Kh, Bahaadinbeigy K, Ghaffaripour Z, Ghaemi MM. The Design and Evaluation of a Mobile based Application to
Facilitate Self-care for Pregnant Women with Preeclampsia during COVID-19 Prevalence. J Biomed Phys Eng. 2021;11(4):551-560. Facilitate Self-care for Pregnant
doi: $10.31661 /$ jbpe.v0i0.2103-1294

Keywords

Pregnancy; COVID-19; Pre-Eclampsia; Mobile Applications; Self-Care

\section{Introduction}

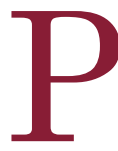
reeclampsia is one of the most fear-provoking complications in pregnancy, which often occurs as hypertension and proteinuria during the third trimester. This complication can progress rapidly and cause serious complications such as maternal and fetal death [1]. The emergence of preeclampsia is often associated with high blood pressure and various organ failures such as kidney, liver and lung dysfunctions [2]. Since in pregnancy, mothers' immune and cardiopulmonary systems
${ }^{1} \mathrm{PhD}$ Candidate, Student Research Committee,

Department of Health

Information Manage-

ment, Kerman University

of Medical Sciences,

Kerman, Iran

${ }^{2} \mathrm{MD}$, PhD, Associate

Professor of Medical

Informatics. Medical

Informatics Research

Center, Institute for Fu-

tures Studies in Health,

Kerman University of

Medical Sciences, Ker-

man, Iran

${ }^{3} \mathrm{MSc}$ student, School of

Health Management and

Information Sciences,

Iran University of Medi-

cal Sciences, Tehran,

Iran

${ }^{4} \mathrm{PhD}$, Department of

Health Information

Management, Kerman

University of Medical Sci-

ences, Kerman, Iran

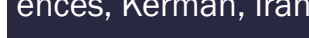

Corresponding author:

Mohammad Mehdi

Ghaemi

Department of Health

Information Manage-

ment, Kerman University

of Medical Sciences,

Kerman, Iran

E-mail: dr.mghaemi@

gmail.com

Received: 29 March 2021 Accepted: 10 June 2021 
undergo physical changes, managing and controlling preeclampsia have become even more challenging during the COVID-19 pandemic as if these mothers become infected by respiratory viruses like COVID-19 and thus their preeclampsia will worsen [3]. Using self-care guidelines can be a promising solution to prevent or overcome respiratory diseases, especially COVID-19 [4].

Pregnancy self-care has been defined as the observance of principles and care programs, maintaining maternal and fetal health during pregnancy, childbirth, and the postpartum period [5]. Self-care activities can reduce the risk of Covid 19 and improve the quality of life [6]. Mobile phones are one of the most salient technological devices that have offered self-care services to pregnant women during the pandemic. Over the recent years, mobile technologies have been widely used to provide and/or receive pregnancy healthcare services [7]. Many people use smartphones as a primary means to access healthcare information and manage their health. During their pregnancy and postpartum period, pregnant women increasingly rely on social media and mobile applications to access informative resources and health services that would facilitate self-care and infant care processes for them [8]. Besides instant accessible healthcare information, mobile health applications improve interactions with the healthcare system [7]. These programs provide reliable accurate information to their users whenever and wherever they need, manage users' health and promote a healthy lifestyle [8]. Self-care applications have removed spatial and temporal barriers that most healthcare providers encounter when attempting to offer quality services [9].

To our knowledge, no study has yet been conducted to design a proper mHealth application, facilitating self-care for pregnant women, who suffer from preeclampsia in the COVID-19 pandemic. Therefore, the aim of this study was to design, develop, and evaluate such an application, leading to proper useful self-care guidelines for pregnant women.

\section{Material and Methods}

This study is conducted based on two steps, as follows:

\section{Information needs and capabilities re- quired to design the application}

In the first stage, the necessary information and feature needed in designing a mobilebased application of self-care for pregnant women, who suffer from preeclampia during COVID-19 prevalence, were investigated. The research sample at this stage consisted of two groups of obstetricians and pregnant women(with/without preeclampsia,and with/ without COVID-19 infection). In the group of physicians, all 17 physicians working in hospitals and medical centers affiliated to Kerman University of Medical Sciences were invited to participate in the study. In the group of pregnant women, 20 people were invited. Ten doctors and 10 pregnant women agreed to participate in the study.

A five-Likert scale questionnaire was designed based on the information gathered from other studies, conducted on perfoming selfcare against COVID-19 [10-15], pre-eclampsia and pregnancy [16-18]. We also used the following online websites to design the questionnaire: World Health Organization (WHO), Obsessive Compulsive Disorder (OCD) foundation, and Centers for Disease Control and Prevention (CDC) [19-21]. By this questionnaire, the opinion of obstetricians and pregnant women about the importance of information needs and data elements needed to design the application was obtained.

The first part of the questionnaire included the participants' demographic information. Other questions were placed in the following four categories: user's profile (11 questions), lifestyle (8 questions), disease prevention and control (24 questions), application capabilities (14 questions), and user's satisfaction (9 questions). The questionnaire designed for pregnant women and physicians was similar. 
The face and content validity of the questionnaire was assessed and confirmed by 6 experts ( 3 medical informatics specialists and 3 obstetricians). The questionnaire was answered by 20 individuals ( 10 obstetricians and 10 pregnant women), and its reliability was 0 . 896 based on Cronbach's alpha.

Between December $16^{\text {th }}$ to $30^{\text {th }}, 2020$, the researcher distributed the questionnairs among the participants and later collected them. Mann-whitney's test was used for data analysis using SPSS software version 23. According to mann-whitney's test, only those educational-informative needs and application capabilities, which had obtained $\mathrm{p}>0.005$, were considered in designing the application.

\section{Design and evaluation the application prototype}

In the second stage, based on the required information and expected capabilities that were determined and approved in the first stage, a mobile-based application was designed. The application was designed via Java programming language in an Android Studio programming environment. After designing the application, its usability was assessed to detect any possible issues. To complete this stage, 20 pregnant women were invited to share their opinions, from which 15 accepted to participate in the evaluation.

To perform the evaluation, firstly the application was installed on the participants' mobile devices. Secondly, the participants were asked to use the application over the next ten days. After that, participants were asked to express their opinions about the application, later assessed via Questionnaire for User Interaction Satisfaction (QUIS) (version 5.5) [22].

By reference to previous studies, the validity and reliability (0.94) of this questionnaire were confiremd validity [23]. The QUIS questionnaire here had six categories:1-the participants' demographic information, 2-the overal reaction to the app, 3-the screen (4 questions), 4-the terminology and information used in application, 5-the learning, and finally the 6-the application capabilities. This questionnaire was designed based on the 10-point Likert scale. Scores 0-3 in this questionnaire were classified as poor, 3. 1-6 as intermediate, and 6. 1-9 as good. The results obtained at this stage were analyzed by means of descriptive statistics in SPSS software version 23, where means and standard deviation were also calculated.

\section{Technical presentation}

The results from both previous steps are presented below.

\section{Information needs and capabilities re- quired to design the application}

Most participants (pregnant women's and obstetrician) in this phase were between 41-50 years old and had between 6-11 years of work experience. In pregnant women's group, 4 participants $(40 \%)$ were infected by COVID-19 and $3(30 \%)$ were infected by preeclampsia. Also, $50 \%$ of these participants had one or two previous pregnancies.

The following 5 categories were considered in identifying information needs and determining application capabilities: User's profile, lifestyle, disease prevention and control, application capabilities and user's satisfaction. Table 1 demonstrates the participants' opinions on the necessity of considering these elements in designing the application. According to Table 1, the user's profile was the only category whose eight data elements (i. e. national ID number, age, weight, height, education, occupation, address, and contact number) earned a $p$-value $<0.05$. These data elements were not considered in the application design.

Among all 66 considered elements, all 58 data elements in 5 categories with a p-value $>0.05$ were considered in designing the application. Eight data elements were also removed.

2. Design and evaluation the application prototype

In the second stage of this study, a self-care application was designed based on the ob- 
Khadijeh Moulaei, et al

Table 1: The participants' expectations of the application in terms of its information and capabilities

Data Elements

From both pregnant women and obstetritcian's perspectives

\begin{tabular}{|c|c|c|}
\hline & & p-value \\
\hline \multirow{11}{*}{ 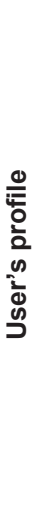 } & Name and surname & 0.77 \\
\hline & National ID number & 0.02 \\
\hline & Age & 0.02 \\
\hline & Weight & 0.04 \\
\hline & Height & 0.01 \\
\hline & Education & 0.04 \\
\hline & Occupation & 0.04 \\
\hline & Address & 0.03 \\
\hline & Contact number & 0.02 \\
\hline & History of underlying diseases & 0.58 \\
\hline & History of having preeclampsia & 0.59 \\
\hline \multirow{8}{*}{ 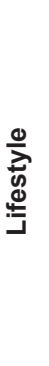 } & Physical exercise & 0.93 \\
\hline & Sleep & 0.24 \\
\hline & Proper nutrition and a healthy diet during the COVID-19 pandemic & 0.67 \\
\hline & Mental and spiritual health during pregnancy & 0.25 \\
\hline & Family and family interactions & 0.56 \\
\hline & Smoking, hookah and alcohol consumption & 0.07 \\
\hline & Pregnant women's stress management & 0.07 \\
\hline & Emotional relations & 0.24 \\
\hline \multirow{24}{*}{ 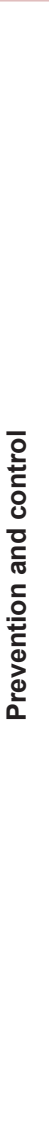 } & Introducing and explaining COVID-19 & 0.06 \\
\hline & preeclampsia symptoms & 0.06 \\
\hline & Preeclampsia complications & 0.31 \\
\hline & COVID-19 symptoms & 0.20 \\
\hline & COVID-19 complications & 0.35 \\
\hline & COVID-19 transmission from mother to fetus & 0.63 \\
\hline & COVID-19 and preeclampsia during pregnancy and lactation & 0.53 \\
\hline & COVID-19 prevention & 0.15 \\
\hline & Preeclampsia prevention & 0.36 \\
\hline & Personal hygiene & 0.38 \\
\hline & Smoking, hookah and alcohol consumption during pregnancy and COVID-19 & 0.85 \\
\hline & Proper methods for connecting with others & 0.95 \\
\hline & Harmful aspects of using prevention methods like wearing masks, using sanitizers. & 0.28 \\
\hline & methods to avoid stressful COVID-19 environments and relations & 0.94 \\
\hline & Maintaining proper nutrition and a healthy diet during COVID-19 prevalence & 0.21 \\
\hline & Maintaining proper nutrition and a healthy diet when having preeclampsia & 0.25 \\
\hline & Introducing methods to maintain mental health when infected by COVID-19 or having preeclampsia & 0.41 \\
\hline & Primary measures to be performed when infected by COVID-19 or having preeclampsia & 0.99 \\
\hline & Introducing methods to reduce or maintain weight during pregnancy & 0.27 \\
\hline & Performing regular pregnancy examinations & 0.24 \\
\hline & Stressing the significance of pregnancy care & 0.37 \\
\hline & Home quarantine & 0.27 \\
\hline & Obtaining reliable news and information & 0.68 \\
\hline & Regular use of medicine & 0.61 \\
\hline
\end{tabular}


Preeclampsia Self-care App during the COVID-19 Prevalence

\begin{tabular}{|c|c|c|}
\hline Categories & Data Elements & $\begin{array}{l}\text { From both pregnant } \\
\text { women and obstetri- } \\
\text { tcian's perspectives }\end{array}$ \\
\hline & & p-value \\
\hline \multirow{14}{*}{ 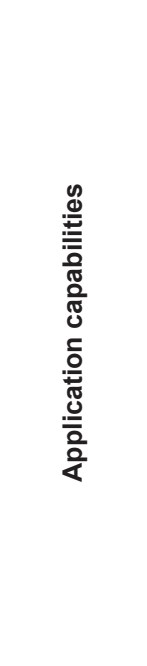 } & Introducing specialized COVID-19 medical centers & 0.77 \\
\hline & Ability to search the location of medical centers and physicians' offices & 0.90 \\
\hline & Managing medication intake & 0.92 \\
\hline & Stressing the significance of regular use of medicine & 0.35 \\
\hline & Ability to record medical allergies & 0.11 \\
\hline & Self-assessment & 0.16 \\
\hline & Stress reduction and control & 0.95 \\
\hline & Nutrition and diet management & 0.44 \\
\hline & Sleep management & 0.55 \\
\hline & Contacting physicians & 0.53 \\
\hline & Doctor's appointment reminder & 0.59 \\
\hline & Connecting with other users & 0.93 \\
\hline & Searching through available educational material & 0.41 \\
\hline & Application settings & 0.77 \\
\hline \multirow{9}{*}{ 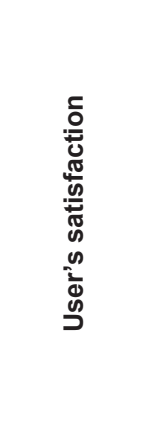 } & Avoiding the display of massive information in one single page & 0.50 \\
\hline & Avoiding the use of small icons & 0.66 \\
\hline & Ensuring well readability of text on the display & 0.56 \\
\hline & Use of magnifying capability & 0.53 \\
\hline & Use of transparent terms and phrases to facilitate user's tasks & 0.47 \\
\hline & Proper arrangement of application information & 0.15 \\
\hline & Position of appearing messages on the display & 0.62 \\
\hline & Providing alerts to record essential information & 0.17 \\
\hline & Relating the content of messages to the performed tasks & 0.04 \\
\hline
\end{tabular}

tained results from the needs assessment performed in stage one (Figure 1). In the user's profile category, users record their demographic and clinical information. The lifestyle category offers self-care recommendations to the user. In "Disease prevention and control", in addition to introducing and explaining COVID-19 to pregnant women, suffering from preeclampsia, different prevention and controlling methods are provided to users based on specialists' recommendations and reliable scientific information in order to help them learn how to avoid and/or manage COVID-19 and preeclampsia by preforming proper selfcare and self-management processes.

The "Application capabilities" category consists of 14 data elements, which are described below.

When specialized medical centres are intro- duced, a database has been set in this application, containing a list of the medical centers and doctors that are available in each province. Users can easily access their desired information by searching the province, city or doctor's name.

In "managing medication intake" and "stressing the significance of regular medication intake" (Figure 2), it is possible to record the user's medications, their doses, and time of intake. Also, setting reminders, viewing a list of previous reminders and medications, and accessing educational videos on regular medication intake are possible. Here, the users can record their medical allergies and list all the medications to which she has shown an allergic reaction. This will also cause her to provide this information to her physician if necessary. In "self-assessment", users can record 


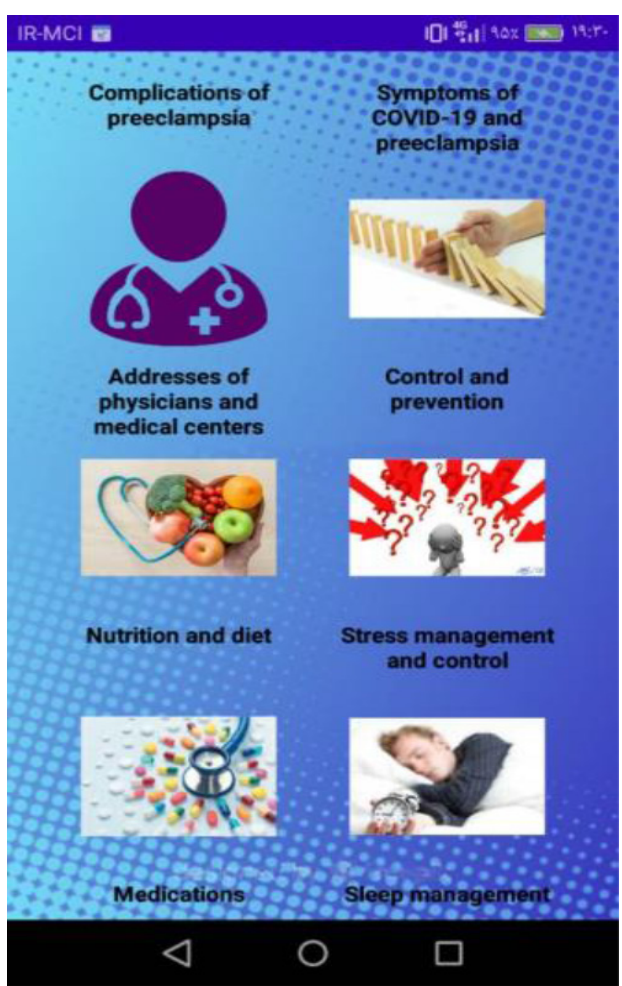

Figure 1: The main page of the self-care application for pregnant women with preeclampsia against COVID-19.

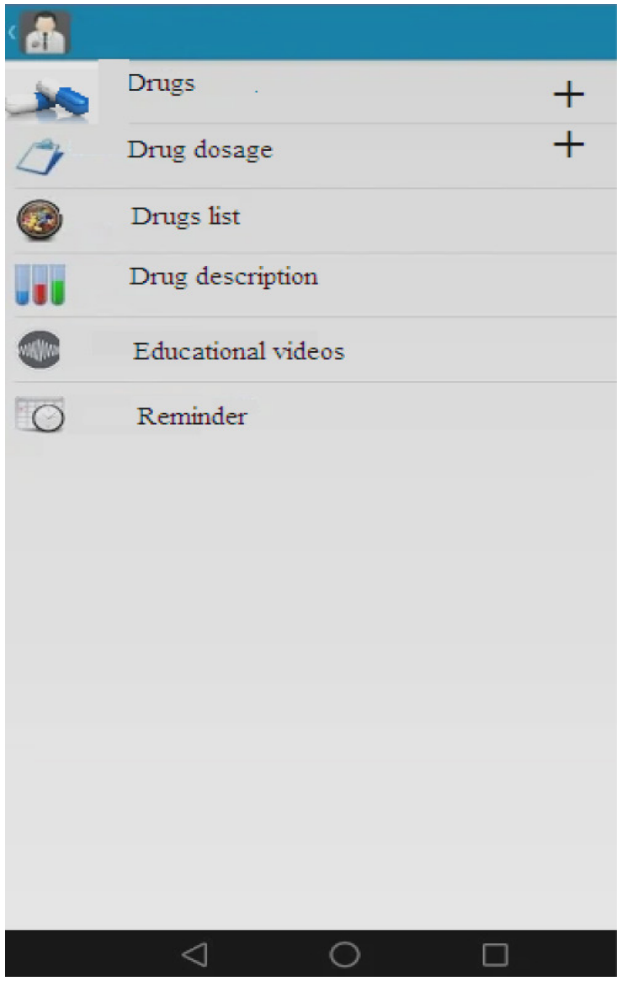

Figure 2: Managing medication intake the severity of their side effects along with the time and date of occurrence. They can keep a history of previous self-assessments to present later to their physician as well. In "stress reduction and control", users can mark their stress level by selecting one of the low, medium and high stress level options in the application. By selecting the medium or high level, educational videos related to stress reduction and control, relaxing music and hopeful messages and strategies for overcoming stress are provided to the user.

Users are able to record accurate information about their physical conditions in the "nutrition and diet management" section, which covers areas such as the users' daily diets, main meals, used snacks, consumed calories, and the exact time of food intake. This will help them to better track and control their weight. After recording relevant information, the user will receive reminders and messages for timely consumption and weight loss and control (Figure 3). In "sleep management", the

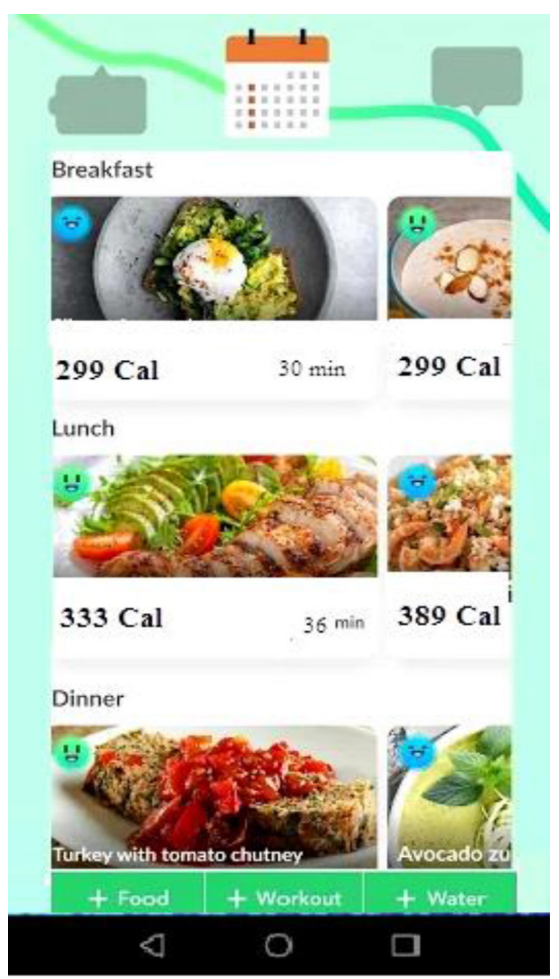

Figure 3: Nutrition and diet management 
user can record her wake up time and plan a proper and stress-free sleep pattern at different time intervals. Also, some helpful videos and materials are provided here to signify the benefits of getting adequate sleep.

In "contacting physicians", a list of doctors, the locations of their offices and contact information are provided. In "doctor's appointment reminder", the user is able to record the exact time of her doctor's appointment, the doctor's name, the location of the medical center, and also a description of the appointment. Based on the recorded date, periodic reminders are given to the user. In "connecting with other patients", users can join a group on WhatsApp or Telegram, where they can discuss different issues and interact. Here, a number of physicians volunteer for group admins in order to provide helpful services to pregnant mothers. In "searching educational material", users can search and retrieve the information they need in the application. In "application settings", users can change some default settings such as the font, size and color of text.

Finally, in the "user's satisfaction" category, pregnant women expressed their positive opinion about the 9 data elements. They believed that these data elements would make the application easy and consistent to use.

In the application evaluation stage, user's satisfaction with the designed self-care application and its applicability were assessed. In this phase, most pregnant women were 20-30 years old, and had a bachelor's degree that 6 were infected by COVID-19 and 2 had preeclampsia at the time of conducting this study. Table 2 shows women's opinions on self-care application usability and user's satisfaction. As observed, an average above 6 was obtained in all assessed dimensions. Thus, the users believed that the usability of the application was at a "good" level.

\section{Discussion}

In the present study, a mobile-based application was designed and evaluated for self-
Table 2: Assessing self-care application usability and user's satisfaction

\begin{tabular}{cc} 
Assessed aspects & Mean (士SD) \\
\hline Overall reaction to the app & $7.12( \pm 1.46)$ \\
\hline Screen & $7.32( \pm 1.55)$ \\
\hline $\begin{array}{c}\text { Terminology and information used } \\
\text { in application }\end{array}$ & $7.56( \pm 1.34)$ \\
\hline Leaning & $7.87( \pm 0.99)$ \\
\hline App capabilities & $7.43( \pm 0.78)$ \\
\hline Total & $8.30( \pm 1.27)$
\end{tabular}

care of pregnant women suffering from preeclampsia during the COVID-19 pandemic. Obstetricians and pregnant women, who had participated in this study, had confirmed all data elements related to user's lifestyle, disease prevention and control, application capabilities, and user's satisfaction. Also, the user's profile was the only section whose eight data elements, i. e. national ID number, age, weight, height, education, occupation, address, and contact number were not considered in the application design. Out of the total of 66 data elements, divided into 5 categories, 58 were considered in designing the application.

In the present study, as in other studies, the essential information and required capabilities related to self-care were presented in the following 5 categories: user's profile, lifestyle, disease prevention and control, application capabilities and user's satisfaction. In a study conducted by Saeidnia et al. [24], they classified the needs of their self-care system into the following four categories: "demographic information, clinical needs, self-care strategies and technical characteristics". They did not however, design or assess a certain application in their study and their focus was merely on conducting an assessment to design applications, concentrated on COVID-19 without considering any other diseases. Chaudhry et al. [25] designed, developed and later assessed an application for low-income pregnant women. In this study, features such as tracking referrals, data sharing among service providers, 
calendars and reminders for pregnant women, coordination with prenatal care coordinators (PNCCs), trackers for specific health indicators and an educational library were used. Nine pregnant women used the Computer System Usability Scale (CSUS) questionnaire to assess its usability [25]. Sajjad et al. [26] designed the +Baby application to help and support pregnant women in Pakistan. By the use of this application, women could record their health history by means of different charts, record their prayers, a section on prayers, Quranic chapters and verses, keep track of their weight and daily food intake for example the sufficient amount of fruit and vegetables consumed per day, find answers to local myths, misconceptions, and Islamic FAQs, use a physical exercise program (recommended by a gynecologist based on the woman's condition and stage of pregnancy), find instructions on how to perform the exercises, use a push notification system to do different tasks, use a baby tracker; and review a quick guide (tips) helping pregnant women to avoid risky behaviors. Fourteen pregnant women evaluated the usability of +Baby through interviews [26].

Some studies have emphasized the significance of self-care during pregnancy and/or during the COVID-19 pandemic. These studies, however, have not specifically focused on designing, developing, and assessing a selfcare application for pregnant women. McIntyre [5] stated that taking self-care measures during pregnancy can ensure maternal and fetal health during pregnancy, childbirth, and postpartum period. Pregnant women should take care of themselves during their pregnancy to ensure their own health and that of their babies. Moradi et al. [11] evaluated the use of self-care strategies by women with gestational diabetes mellitus (GDM) during COVID-19. Their findings showed that following selfcare programs can improve maternal and fetal health status in women suffering from GDM. Moreover, appropriate self-care guidelines can be considered as practical approaches to reduce the spread of COVID-19 and improve the quality of life.

All of the abovementioned studies have emphasized on the significance of performing self-care during pregnancy and/or during the COVID-19 outbreak. None of these studies, however, have designed and developed a selfcare application for pregnant women to protect them against COVID-19. To sum, these studies have investigated issues like the benefits of performing self-care during pregnancy [5], the nature of pregnancy-related applications [27], the effects of education on improving self-care processes [28], self-care strategies for women with GDM during the COVID-19 pandemic [11], and/or self-care guidelines for individuals, infected by COVID-19 without any need to hospitalization [4].

Given the above studies, none of the studies have focused on designing, developing, and evaluating a mobile-based application for self-care of pregnant women during $\mathrm{CO}$ VID-19. Although there is a incremental need and desire to use mHealth applications to support pregnant women, but these needs have not been well considered by governments, organizations and health centers. Self-care guidelines can be a useful solution to prevent COVID-19 and manage its complications [4]. These self-care guidelines will be more effective for users when they are provided through technology. Various technologies, especially mobile-based technologies, are usually created to elevate public knowledge and improve their self-care and self-management abilities. When public knowledge increases and selfcare and/or self-management skills improves, a change will occur in the public's lifestyle and behavior patterns, resulting in an eventual improvement of the health status.

The limited number of participants, in the needs assessment and evaluation phase and the short time to evaluate the application (ten days), were the limitations of this study. It is recommended that future studies should be carried out on a larger number of participants 
during a longer time span. Another limitation of the study was that the designed application could only be installed on mobiles with Android operating systems. Applications can be designed to be installed on other mobile operating systems (e. g. iOS). Therefore, they will be used by more users.

\section{Conclusion}

In this study, a mobile-based application to facilitate self-care in pregnant women, suffering from preeclampsia during the COVID-19 pandemic, was designed, developed and evaluated. By providing different services, options and capabilities for the user, the application helps to reduce pregnant women stress. The application can also increase users' knowledge and better their attitude towards COVID-19 and preeclampsia. When pregnant mothers' knowledge and attitude is improved, their selfcare and self-management processes will also better, resulting in ultimately a less challenging pregnancy and delivery. The application will also reduce frequent personal attendances to doctors' offices, reduce physician's workload, save their time, and reduce the possible risk of becoming infected by the COVID-19 for both patients and physicians.

\section{Acknowledgment}

The authors would like to thank all experts who freely participated in this study.

\section{Conflict of Interest}

\section{None}

\section{References}

1. Rana S, Lemoine E, Granger JP, et al. Preeclampsia: Pathophysiology, Challenges, and Perspectives. Circ Res. 2019;124(7):1094-112. doi: 10.1161/CIRCRESAHA.118.313276. PubMed PMID: 30920918.

2. Bokslag A, Van Weissenbruch $\mathrm{M}, \mathrm{Mol} B W$, et al. Preeclampsia; short and long-term consequences for mother and neonate. Early Hum Dev. 2016;102:47-50. doi: 10.1016/j.earlhumdev.2016.09.007. PubMed PMID: 27659865.
3. Azarkish F, Sheikhi F, Mirkazehi Z, et al. Preeclampsia and the crucial postpartum period for Covid-19 infected mothers: A case report. Pregnancy Hypertens. 2021;23:136-9. doi: 10.1016/j. preghy.2020.10.012. PubMed PMID: 33388729. PubMed PMCID: PMC7604163.

4. Mehraeen E, Hayati B, Saeidi S, et al. Self-care instructions for people not requiring hospitalization for coronavirus disease 2019 (COVID-19). Arch Clin Infect Dis. 2020;15(COVID-19):e102978. doi: 10.5812/archcid.102978.

5. McIntyre $P$, et al. Pregnant adolescents: delivering on global promises of hope. World Health Organization; 2006. p. 2-28.

6. Waris Nawaz M, Imtiaz S, Kausar E. Self-care of Frontline Health Care Workers: During COVID-19 Pandemic. Psychiatr Danub. 2020;32(3-4):55762. doi: 10.24869/psyd.2020.557. PubMed PMID: 33370766.

7. Riegel B, Jaarsma $T$, Lee CS, et al. Integrating Symptoms In to the Middle-Range Theory of Self-Care of Chronic Illness. ANS Adv Nurs Sci. 2019;42(3):206-15. doi: 10.1097/ ANS.0000000000000237. PubMed PMID: 30475237. PubMed PMCID: PMCID: PMC6686959.

8. Chan KL, Chen M. Effects of Social Media and Mobile Health Apps on Pregnancy Care: Meta-Analysis. JMIR Mhealth Uhealth. 2019;7(1):e11836. doi: 10.2196/11836. PubMed PMID: 30698533. PubMed PMCID: PMC6372934.

9. Lee Y, Moon M. Utilization and Content Evaluation of Mobile Applications for Pregnancy, Birth, and Child Care. Healthc Inform Res. 2016;22(2):7380. doi: 10.4258/hir.2016.22.2.73. PubMed PMID: 27200216. PubMed PMCID: PMC4871848.

10. Turner-McGrievy GM, Beets MW, Moore JB, et al. Comparison of traditional versus mobile app self-monitoring of physical activity and dietary intake among overweight adults participating in an mHealth weight loss program. $J \mathrm{Am}$ Med Inform Assoc. 2013;20(3):513-8. doi: 10.1136/ amiajnl-2012-001510. PubMed PMID: 23429637. PubMed PMCID: PMC3628067.

11. Moradi F, Ghadiri-Anari A, Enjezab B. COVID-19 and self-care strategies for women with gestational diabetes mellitus. Diabetes Metab Syndr. 2020;14(5):1535-9. doi: 10.1016/j. dsx.2020.08.004. PubMed PMID: 32947751. PubMed PMCID: PMC7837010.

12. Yang Z, Wang M, Zhu Z, et al. Coronavirus disease 2019 (COVID-19) and pregnancy: a systematic review. J Matern Fetal Neonatal Med. 2020:1-4. 
Khadijeh Moulaei, et al

doi: 10.1080/14767058.2020.1759541. PubMed PMID: 32354293.

13. Chen H, Guo J, Wang C, et al. Clinical characteristics and intrauterine vertical transmission potential of COVID-19 infection in nine pregnant women: a retrospective review of medical records. Lancet. 2020;395(10226):809-15. doi: 10.1016/S01406736(20)30360-3. PubMed PMID: 32151335. PubMed PMCID: PMC7159281.

14. Kazemi-Arpanahi H, Moulaei K, Shanbehzadeh M. Design and development of a web-based registry for Coronavirus (COVID-19) disease. Med J Islam Repub Iran. 2020;34:68. doi: 10.34171/ mjiri.34.68. PubMed PMID: 32974234. PubMed PMCID: PMC7500427.

15. Liang $H$, Acharya $G$. Novel corona virus disease (COVID-19) in pregnancy: What clinical recommendations to follow? Acta Obstet Gynecol Scand. 2020;99(4):439-42. doi: 10.1111/aogs.13836. PubMed PMID: 32141062.

16. Panahi L, Amiri M, Pouy S. Risks of Novel Coronavirus Disease (COVID-19) in Pregnancy; a Narrative Review. Arch Acad Emerg Med. 2020;8(1):e34. PubMed PMID: 32232217. PubMed PMCID: PMC7092922.

17. Coronado-Arroyo JC, Concepción-Zavaleta MJ, Zavaleta-Gutiérrez FE, et al. Is COVID-19 a risk factor for severe preeclampsia? Hospital experience in a developing country. Eur J Obstet Gynecol Reprod Biol. 2021;256:502-3. doi: 10.1016/j. ejogrb.2020.09.020. PubMed PMID: 32958322. PubMed PMCID: PMC7489262.

18. Rolnik DL. Can COVID-19 in pregnancy cause pre-eclampsia? BJOG. 2020;127(11):1381. doi: 10.1111/1471-0528.16369. PubMed PMID: 32570284. PubMed PMCID: PMC7361765.

19. World Health Organization. Self care during COVID-19. WHO; 2020 [cited 2020 June 16]. Available from: https://www.who.int/news-room/photo-story/photo-story-detail/self-care-during-covid-19.

20. Centers for Disease Control and Prevention. Coping with Stress. CDC; 2020 [cited 2020 June 16]. Available from: https://www.cdc.gov/ coronavirus/2019-ncov/daily-life-coping/managing-stress-anxiety.html.

21. International OCD Foundation. Obsessive Compulsive Disorder. OCD: 2020 [cited 2020 July 10].
Available from: https://iocdf.org/covid19/self-careduring-covid-19.

22. Chin JP, Diehl VA, Norman KL. Development of an instrument measuring user satisfaction of the human-computer interface. Proceedings of the SIG$\mathrm{CHI}$ conference on Human factors in computing systems; USA: Association for Computing Machinery; 1988. p. 213-8. doi: 10.1145/57167.57203.

23. Raghunath N, Dahmen J, Brown K, Cook D, Schmitter-Edgecombe M. Creating a digital memory notebook application for individuals with mild cognitive impairment to support everyday functioning. Disabil Rehabil Assist Technol. 2020;15(4):421-431. doi: 10.1080/17483107.2019.1587017. PubMed PMID: 30907223. PubMed PMCID: PMC7314313.

24. Saeidnia H, Mohammadzadeh Z, Saeidnia M, et al. Identifying Requirements of a Self-care System on Smartphones for Preventing Coronavirus Diseas. Iran J Med Microbiol. 2020;14(3):241-51. doi: 10.30699/ijmm.14.3.241.

25. Chaudhry BM, Faust L, Chawla NV. From Design to Development to Evaluation of a Pregnancy App for Low-Income Women in a Community-Based Setting. Proceedings of the 21st International Conference on Human-Computer Interaction with Mobile Devices and Services; USA: Association for Computing Machinery; 2019. p. 1-11. doi: 10.1145/3338286.3340118.

26. Sajjad UU, Shahid S. Baby+ a mobile application to support pregnant women in Pakistan. Proceedings of the 18th International Conference on Human-Computer Interaction with Mobile Devices and Services Adjunct; USA: Association for Computing Machinery; 2016. p. 667-74. doi: 10.1145/2957265.2961856.

27. Tripp N, Hainey K, Liu A, Poulton A, Peek M, Kim J, Nanan R. An emerging model of maternity care: smartphone, midwife, doctor? Women Birth. 2014;27(1):64-7. doi: 10.1016/j.wombi.2013.11.001. PubMed PMID: 24295598.

28. Rezaeean SM, Abedian Z, Latifnejad-Roudsari R, Mazloum SR, Abbasi Z. The effect of prenatal self-care based on orem's theory on preterm birth occurrence in women at risk for preterm birth. Iran J Nurs Midwifery Res. 2020;25(3):242. doi: 10.4103/ijnmr.IJNMR_207_19. PubMed PMID: 32724771. PubMed PMCID: PMC7299423. 